\title{
Noise Reduction and Random Error Modeling of MEMS Gyroscope
}

\author{
Pengjiao Liu, a, Gongliu Yang, , b Suier Wang, c \\ ${ }^{1}$ School of Beihang University, Beijing, 100191, China \\ ${ }^{2}$ Henan Thinker Rail Transportation Research Inc, Zhengzhou, 450001, China

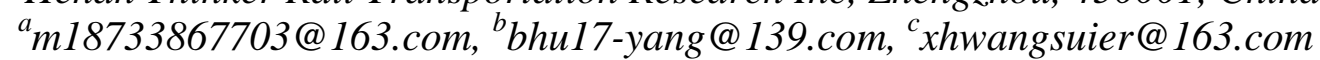

Keywords: adaptive time-scale decomposition, MEMS gyro, AR model, random error.

\begin{abstract}
For the nonlinear, non-stationary and weak correlation signals existing in a MEMS (Microelectronic-mechanic system) gyro, a denosing method based on improved Adaptive Time-scale Decomposition (IATD) was proposed. The signals, which was captured by the static experiment, were decomposed into a cluster of intrinsic time-scale component based on IADT process. Then, according to the characteristics of the gyro random error, the gyro signals were reconstructed to implement the signal denoising. AR (2) model was applied to set up a mathematical model of the reconstructed signals. After filtering and modeling, the random error of gyro is reduced by $83.72 \%$, which means the random error of MEMS gyro is suppressed effectively.
\end{abstract}

\section{Introduction}

Micro electro mechanical system (MEMS) gyro has been widely used in low-cost navigation system for its advantages of small size, low cost, impact resistance and high reliability. [1-2]), However, due to the manufacturing process and other reasons, MEMS gyroscopes have low accuracy and noise compared to other gyroscopes, and are particularly affected by temperature. Therefore, the important error terms and low-frequency noise characteristics are "submerged" in high-frequency noise. Consequently, it is difficult to establish the MEMS gyroscope's random error model by using original output directly. [3]. For the above mentioned, it is necessary to denoise the collected original signals.

At present, for the denoising of gyro, wavelet transform is mainly used. The wavelet denoising methods are divided into three categories [4]. They are: modular maximum reconstruction denoising, spatial correlation denoising, and wavelet domain denoising. Among them, the wavelet threshold denoising method is simple and effective, and widely used [5-8]. The idea is to use the Mallet algorithm to obtain the wavelet coefficients at different scales. By comparing with the preset thresholds, the wavelet coefficients with smaller absolute values are set to zero, while the coefficients with larger absolute values are retained or shrunk. Although the wavelet thresholding method can implement simply, but it requires accurate threshold selection, and even it depends on the variance of the noise. At the same time, the basis of the wavelet in the method is a fixed function, and it is not adaptable for non-stationary and non-linear signals, which cannot reflect the essential characteristics of the changed signal [9].The literature [10] involves compressive sensing 
theory to reconstruct the coefficients of the wavelet to achieve the purpose of noise reduction. However, compared with the wavelet threshold denoising, It caused a greater degree of noise removal, and the compression ratio needs to be selected based on experience. When the selection is inappropriate, there is a possibility that the noise reduction result is inferior to the wavelet threshold. The literature [11] adopts empirical mode decomposition and high-order statistical methods for noise reduction. After processing, the SNR (SIGNAL-NOISE RATIO) of the gyroscope is increased by $5.6 \mathrm{~dB}$. Similarly, the angle random walk coefficient and the zero bias instability are reduced by approximately one order of magnitude. That said, the empirical mode decomposition filter is effective and obvious for nonlinear and non-stationary MEMS gyro signals. In literature [12], the CEEMD method is used and the original signal is decomposed into the sum of several IMFs. The former IMF groups are removed and the remaining are reconstructed. By comparing the Allan variances of the signals before and after the reconstruction, it is shown that the noise after CEEMD is greatly reduced, and the effective information of original signal is retained. However, the literature does not give the reasons for discarding the previous IMF groups.

In this paper, the adaptive time-scale decomposition algorithm is used to decompose the original signal collected by the gyroscope. According to the low-frequency characteristics of the random error of the gyroscope, the decomposed intrinsic time-scale component is analyzed in the frequency domain, and the gyro signal is reconstructed based on the spectral characteristics of the intrinsic time-scale component. Further, the Allan variance coefficients of the reconstructed signal and the acquired signal are compared, which shows that the time-scale decomposition algorithm can effectively denoise. Finally, based on the reconstructed signal, a time series autoregressive AR model was established to achieve accurate prediction and analysis of the random error of the gyroscope.

\section{Spectrum Characteristics of MEMS Gyroscope Signals and Allan Variance}

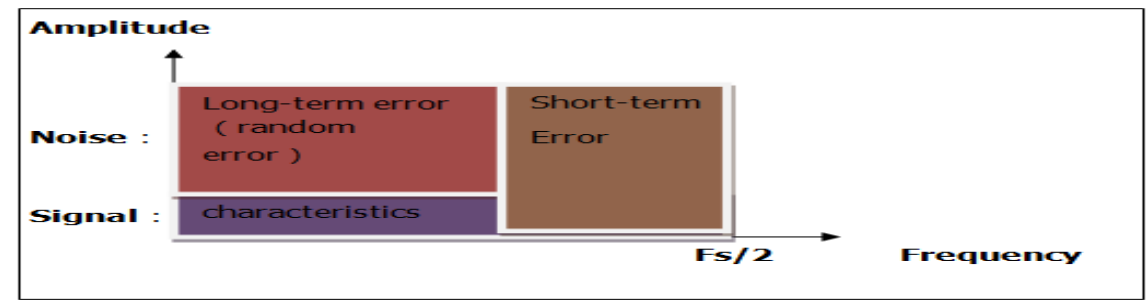

Figure 1. Gyro signal spectral characteristics

According to [13], the spectral characteristics of a gyroscope are shown in Figure 1. The spectrum signal consists of a long-term (random) error, a short-term error, and the gyroscope's own operation characteristics. The short-term error mainly includes the system disturbances (such as the vibration of the carrier) and the associated noise which generates mainly in the high frequency part of the spectrum. While the long-term error is in the low frequency band, which mainly includes the drift error and white noise. The long-term (random) error of a gyroscope can be analyzed by the Allan variance method and eliminated by establishing its predictive model.

In 1966, David Allan proposed a simple method of analysis of variance when studying the stability of oscillators, which is called the Allan variance [14]. The Allan variance is a method to analyze the frequency domain stability in the time domain. It not only can determine the characteristics of the basic random process that produces data noise, but also can identify the source of a given noise item. The relationship between Allan variance and power spectral density is: 


$$
\sigma^{2}(T)=4 \int_{-\infty}^{\infty} S_{\Omega}(f) \frac{\sin ^{4} \pi f T}{(\pi f T)^{2}} d f
$$

Where:

$\sigma 2(\mathrm{~T})$ is the Allan variance;

$\mathrm{S}_{\Omega}(f)$ Is the power spectral density of the random process $\Omega(\mathrm{T})$;

Assuming that the gyro noise sources are statistically independent, the calculated Allan variance is the sum of the squares of the errors for each type. The Allan variance can be obtained by the nonlinear least-squares fitting method. It can be expressed as a polynomial form fitted to a power of -2 to 2 of time t. By solving the coefficients of the polynomial, the coefficients of error sources such as quantization noise, can be determined.

\section{Improved Adaptive Time-scale Decomposition Algorithm}

Adaptive time-frequency analysis is a new type of signal analysis method based on the characteristics of the signal itself. The most representative is the empirical mode decomposition (EMD) proposed by Huang et al. [15] in 1998. The method defines an intrinsic mode function (IMF) with physical meaning of instantaneous frequency. On the basis of its reasonable interpretation, the baseline signal is constructed by averaging the upper and lower extreme point envelopes, so that any complex signal can be decomposed into the sum of several intrinsic mode components. After the method was proposed, EMD has received extensive attention and has been widely used in the field of mechanical fault diagnosis, biomedicine, acoustics, and communications science.However, there is several problems such as over-envelope, under-envelope, modal confusion, and end-point effects.In 2007, Frei et al. [16] proposed the intrinsic time scale decomposition (ITD), which defines the physical rotation of the instantaneous frequency (proper rotation, PR). The baseline signal is given by the transformation of the signal itself. The ITD method can adaptively decompose a complex signal into a number of mutually independent PR components that have physical significance at instantaneous frequencies. Frei et al. conducted a comparative study between the ITD method and the EMD method. The results show that ITD is superior to the EMD method in terms of calculation time, suppression of endpoint effects, and the like. However, the definition of the baseline in the ITD method is based on the linear transformation of the signal itself, so from the second component, There is a noticeable signal distortion.

For the problem of signal distortion, this paper proposes an improved adaptive time-scale decomposition algorithm. The main reason for the signal distortion is that the baseline curve obtained through the linear transformation is not continuous. Therefore, it is necessary to use a curve fitting method to generate baselines. The improved fitting method is based on the combination of cubic spline difference and cubic polynomial difference. Although the cubic spline interpolation method has an ideal smoothness and has second-order continuous differentiability, it will cause "overshoot" phenomenon and produce an extreme point that does not exist. The degree of cubic polynomial interpolation is less smooth than cubic spline. For this purpose, a hybrid method combining cubic spline interpolation and cubic polynomial interpolation is proposed. The calculation process is as follows.

Taking the original signal $x(t)$ as the signal to be processed, it is determined that all local extreme $\left(\tau_{0}, X_{0}\right)$ of the signal $(\mathrm{k}=1,2,3 \ldots \mathrm{M}, \mathrm{M}$ is the number of extreme points). Mirror symmetric continuation methods are used for extending sequence endpoints to obtain the left and right endpoint extremes $\left(\tau_{0}, X_{0}\right)$ and $\left(\tau_{M+1}, X_{M+1}\right)$, and calculate $L_{k+1}$ : 


$$
L_{k+1}=\alpha\left[X_{k}+\frac{\left(\tau_{k+1}-\tau_{k}\right)}{\left(\tau_{k+2}-\tau_{k}\right)}\left(X_{k+2}-X_{k}\right)\right]+(1-\alpha) X_{k+1} \quad(k=1,2,3 \cdots)
$$

Where:

$0<\alpha<1$, generally 0.5 .

According to a series of $L_{k}$ points obtained by the formula (3), The fitting curve $L_{1}$ was calculated by a cubic spline difference fitting method, and then a cubic polynomial difference method is used to fit the curve $L_{2}$, Finally, the baseline signal $L$ is obtained by combination.

$$
L=\beta L_{1}+(1-\beta) L_{2}
$$

Where:

$0<\beta<1$, generally 0.4 , when used;

The fitted curve can not only effectively avoid the "overshoot" phenomenon, but also maintain the advantages of the cubic spline interpolation method. When compared, we conclude that the smoothness of the curve fitted using the combination method is better than the baseline obtained by the linear transformation. Therefore, using a combination of fitting methods to construct the baseline can significantly reduce signal distortion.

The baseline signal $L(t)$ is subtracted from the original signal $x(t)$ to obtain the residual signal $m(t)$ :

$$
m(t)=x(t)-L(t)
$$

Two basic conditions for detecting whether $m(t)$ satisfies the intrinsic time-scale component:

(1) In the entire data sequence, the number of local extreme points $\mathrm{Nm}$ and the number of zero crossing points $N_{z}$ must be equal, or at most one difference, that is, satisfy:

$$
\left|N_{m}-N_{z}\right| \leq 1
$$

It guarantees that the signal satisfies the distribution of Gauss stationary process and removes riding waves.

(2) The baseline signal $L$ has a mean value of 0 because the baseline signal $L$ is the mean of $x(t)$. This condition can force the baseline signal to be symmetrical and smooth the asymmetry amplitude. In actual operation, a small amount of $\varepsilon$ can be set:

$$
\left|\frac{1}{n} \sum_{i=1}^{n} L(i)\right| \leq \varepsilon
$$

It can be considered that the baseline signal has an average of 0 ; If it is not satisfied, take $m(t)$ as the original signal and return to step 1. If it is satisfied, the corresponding intrinsic time scale component $\operatorname{ITC}_{k}=m(t)(k=1,2,3 \ldots)$ is decomposed, and then the signal to be processed is subtracted from the intrinsic time scale component to obtain the remaining signal,

$$
r_{k}(t)=x(t)-I T C_{k}
$$

Let $x(t)=r_{k}(t)$ and rejoin step 1 for calculation. Then, the original signal can be decomposed the original signal into a series of time-scale components. which is: 


$$
x(t)=\sum_{i=1}^{n} I T C_{k}+r_{n}
$$

In order to prevent over-screening due to overly restrictive conditions for the local symmetry of single-mode components, similar to the EMD method, the standard deviation SD between two successive processing results is defined as:

$$
S D=\sum_{i=1}^{n} \frac{\left|m_{k}(i)-m_{k-1}(i)\right|^{2}}{m_{k-1}(i)^{2}}
$$

According to the characteristics of the gyro signal, $S D=0.2$ is taken as the termination condition, that is, $S D \leq 0.2$, then the decomposition is stopped.

The improved adaptive time-scale decomposition algorithm takes the time span corresponding to any two adjacent local extreme points in the signal as the characteristic time-scale parameter. First, the small fluctuation components of the signal with the characteristic time scale are separated. With the increase of the number of decompositions, the characteristic time scale parameter of the residual signal increases until the characteristic time scale parameter is greater than the length of the signal itself. From this it can be seen that the frequency of the intrinsic time scale components decomposed is ranked from high to low.

\section{Experimental Results and Analysis}

In this experiment, MEMS uses the ADIS16488 inertial sensor integrated chip. The experimental data source is a static experiment of 8 hours at a temperature of $25^{\circ}$ on a stationary stand, and the data collection interval is 0.02 seconds, with a total of 1.44 million data. After the wild value elimination, trend item extraction, and zero mean processing, the results are as follows.

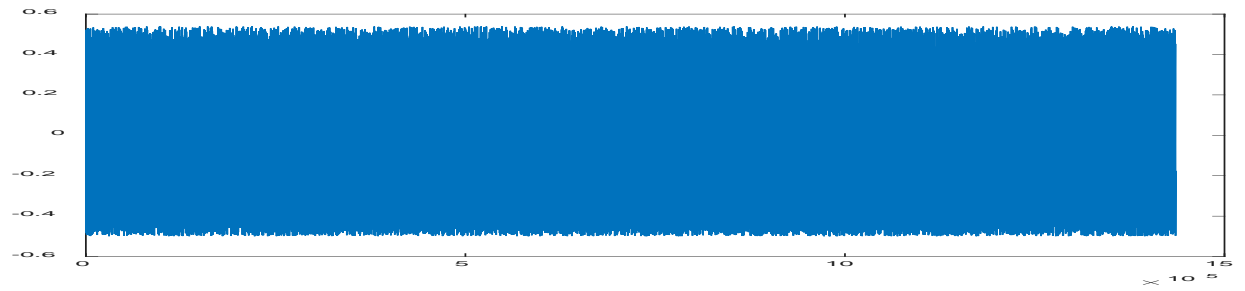

Figure 3. Pre-processing data of MEMS gyroscope static experiment

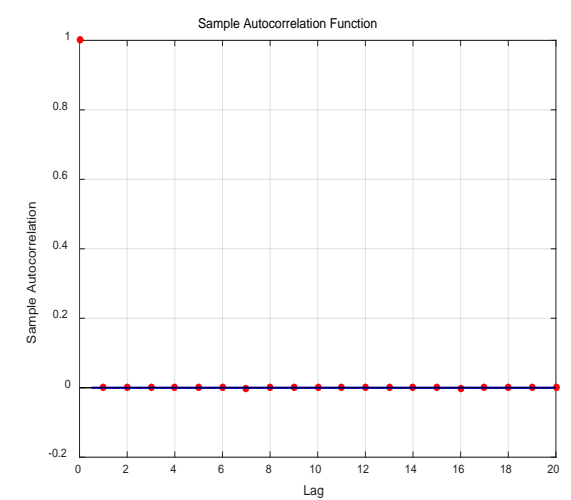

(a) Autocorrelation

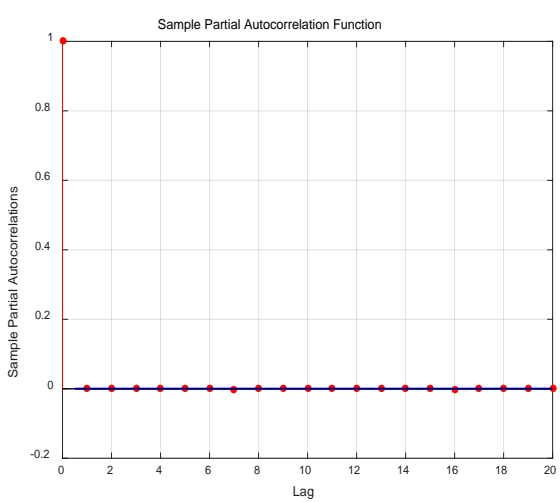

(b) Partial autocorrelation

Figure 4. Correlation test

The run-test method was used to test the stationarity of the preprocessed data. The number of 
positive and negative signs in $x(t)$ was: $N_{1}=717003, N_{2}=717726$. The number of runs is $\gamma=$ 716954 and the calculated test statistic is $U=-1.6021 \in[-1.96,1.96]$. This shows that the preprocessed data is stationary random data. The autocorrelation and partial autocorrelation calculations of the data are as fig. 4

From Figure 4, we can see that the ACF and PACF of the MEMS gyroscope random error sequence all have the first-order truncation characteristic, and it cannot be judged which kind of time series model structure it belongs to. The reason for this phenomenon is that the low-frequency error signal of the MEMS gyroscope is "submerged" in the high-frequency noise, and the correlation of the low-frequency random error sequence of the gyroscope is weakened. Therefore, there is a need for further noise reduction of the data. The improved adaptive time-scale decomposition algorithm is used to decompose the pre-processed signal, and it is decomposed into 11 layers according to the cut-off condition of $S D=0.3$. The decomposition results of $I C T_{1}-I C T_{11}$ are shown in the following figure.

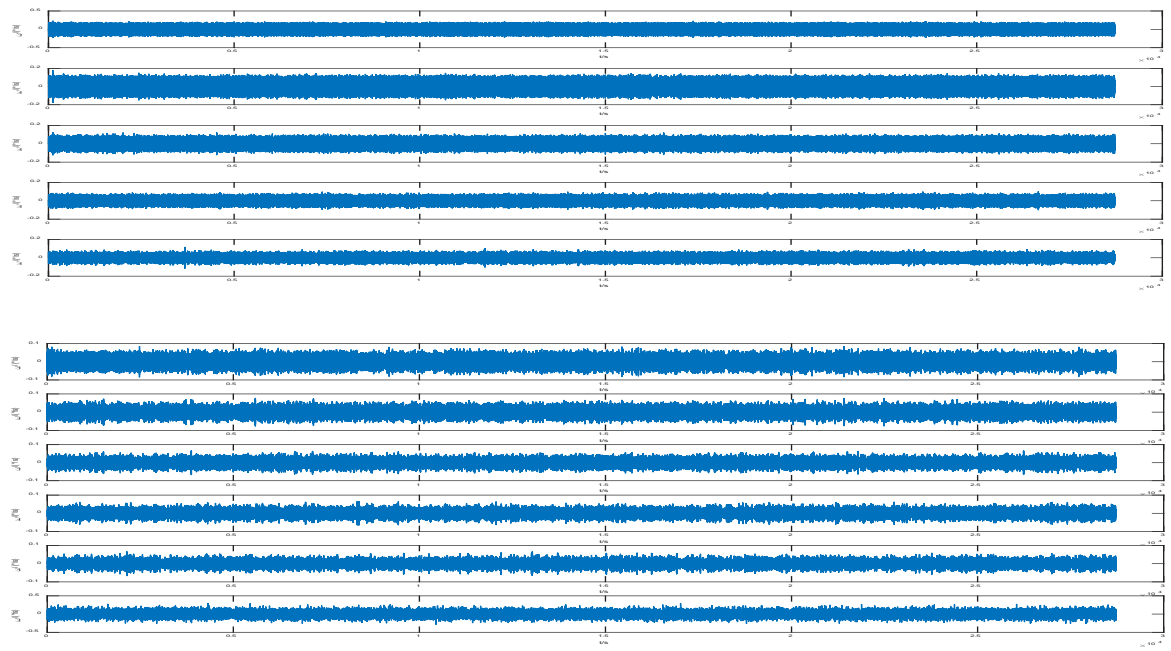

Figure 5.Decomposition results

According to the spectral characteristics of the gyro signal, 11 eigen-time scale components are subjected to spectrum analysis, and from the corresponding spectrum, it can be clearly obtained that the improved adaptive time-scale decomposition algorithm decomposes the processed signal layer by layer from high to low. The $I T C_{1}$ mainly contains high-frequency noise signals and mainly contains the short-term errors of the gyro signals. The $I T C_{11}$ is a low-frequency signal that mainly contains the long-term error of the gyroscope. According to the spectral characteristic of the intrinsic time component, we choose $I T C_{1}-I T C_{11}$ to reconstruct the signal of the gyroscope. AR (2) modeling of the reconstructed signal results as follows:

$$
z(k)=-1.791 z(k-1)+0.8708 z(k-2)+\varepsilon(k)
$$

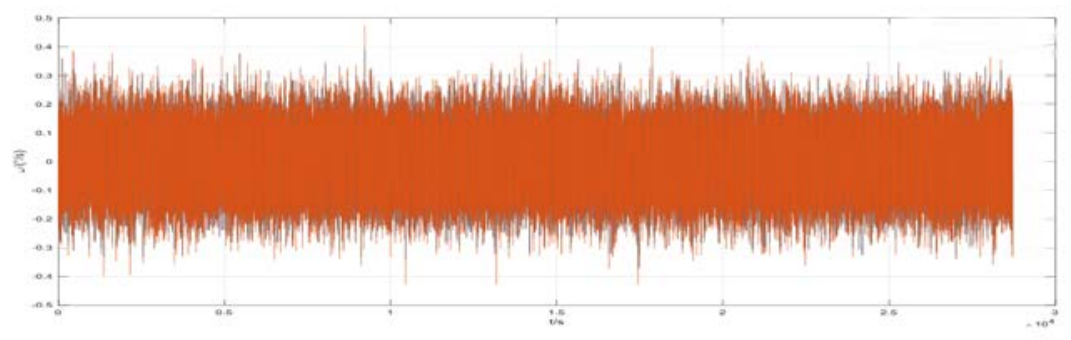

Figure 6. AR(2) modeling of the reconstructed signal results 
As shown in the above figure, the AR(2)-order model can better predict the random error of the gyroscope. As shown in Table 1, the improved adaptive time-scale decomposition reconstructs the signal and the AR(2) model predicts random errors. The Allan variance was used to analyze the original signal and the predicted residuals. The random noise of the gyroscope reduced by an average of $83.72 \%$, among which the angular random walk and the zero bias instability error were the most obvious.

Table 1. Gyroscope random error coefficient statistics before and after processing

\begin{tabular}{ccccc}
\hline $\begin{array}{c}\text { Random error } \\
\text { term }\end{array}$ & Unit & $\begin{array}{c}\text { Before } \\
\text { processing }\end{array}$ & After processing & $\begin{array}{c}\text { Random error } \\
\text { reduction } \\
\text { percentage (\%) }\end{array}$ \\
$\begin{array}{c}\text { Quantization } \\
\text { Error(Q) }\end{array}$ & $\mu \mathrm{rad}$ & $8.4038 \times 10^{-8}$ & $1.2820 \times 10^{-8}$ & 84.74 \\
$\begin{array}{c}\text { Random angle } \\
\text { walk(N) } \\
\begin{array}{c}\text { Zero bias } \\
\text { instability(B) } \\
\text { Angular rate }\end{array}\end{array}$ & $\circ / h^{1 / 2}$ & 0.0201 & 0.00059 & 97.09 \\
$\begin{array}{c}\text { random walk(K) } \\
\text { Scale factor(R) }\end{array}$ & $(/ h) / h^{1 / 2}$ & 0.1282 & 0.0079 & 93.84 \\
\hline
\end{tabular}

\section{Conclusion}

This paper presents an improved adaptive time-scale decomposition algorithm for MEMS gyroscopes. First, the IATD algorithm for nonstationary and nonlinear signals is described. AS the same time, the completeness and adaptability of the algorithm are analyzed. Then, we studied and discussed the collected MEMS static experimental signals. Because the random error signals are submerged in the high-frequency noise signals of the short-term errors of the gyroscopes, the weak correlation of the gyro signals is expressed. . Therefore, the IATD algorithm is used to decompose the original signal of the gyroscope. Spectral analysis is performed on the decomposed signal, and ITC is selected to perform signal reconstruction in accordance with the characteristic frequency of the random error of the gyro. AR (2) was modeled for the reconstructed signal, and the time series model of the random error of the gyroscope was established. Finally, the noise indicators before and after signal processing were compared. The factors such as the angle random walk coefficient and the zero bias instability are reduced by one order, which shows that the combination of the IATD decomposition and the AR (2) model prediction error analysis method has a significant effect on the noise reduction of nonlinear and non-stationary MEMS gyro signals.

\section{References}

[1] Weiping Zhang, Wenyuan Chen, et al. Research progress of MEMS microgyroscope [J]. Micro-nanoelectronics technology, 2011.48(5): 277-285.

[2] Yunhai Zhong, Chengtao Yi. MEMS inertial navigation sensor [J]. Ship Science and Technology, 2014(1): 115-121.

[3] Lijie Zhang. On-line modeling and real-time filtering of multi-criteria MEMS gyro random error[J].Journal of Transduction Technology,2016.29(1):75-79

[4]Quan Pan, Lei Zhang, Jinli Meng, and so on. Wavelet filtering method and application [M]. Beijing: Tsinghua University Press, 2005: 46-48

[5] Li Z, Fan Q, Chang L, et al. Improved Wavelet Threshold Denoising Method for MEMS Gyroscope[C]//11th IEEE International Conference on Control and Automation (ICCA).IEEE 2014:530-534

[6] Meng T, Wang $\mathrm{H}$, Li H, et al. Error modeling and filtering method for MEMS gyroscope[J]. Systems Engineering 
and Electronics, 2009, 8: 038.

[7] Zhang Q, Wang L, Gao P, et al. An innovative wavelet threshold denoising method for environmental drift of fiber optic gyro[J]. Mathematical Problems in Engineering, 2016, 2016.

[8] Shi Y S, Gao Z F. Study on MEMS Gyro Signal De-Noising Based on Improved Wavelet Threshold Method[C]//Applied Mechanics and Materials. Trans Tech Publications, 2013, 433: 1558-1562.

[9] Zhou B, Wang W. Research on a Wavelet Filtering Method of the Fiber Optic Gyroscope Based on EMD[C]//Advanced Materials Research. Trans Tech Publications, 2013, 705: 253-257.

[10]Cheng Cheng, Quan Pan, Shenlong Wang, et al. Research on signal denoising of MEMS gyroscope based on compressed sensing theory. Journal of Instrument and Instrument[J].2012.33(4):769-773

[11] Yandong WANG, Tao ZHANG, Chun-lei YANG, et al. Noise reduction of micromachined gyro based on empirical mode decomposition/high-order statistical method[J]. Optics and Precision Engineering, 2016, 24(3): 574-581.

[12]Ming Zhang, Qingjun Zeng, Xiang Qi, et al. MEMS Gyro Denoising Method for Underwater Vehicle Based on CEEMD[J]. Journal of Transduction Technology, 2014, 27(12): 1622-1626.

[13] Jianjun MA, Zhiqiang ZHENG, Meiping WU. Spectrum Analysis and Noise Reduction Method of MIMU Signal [J]. Editorial Office of Optics and Precision, 2007, 15(2): 261-266. [14] Allan D W. Statistics of atomic frequency standards[J]. Proceedings of the IEEE, 1966, 54(2): 221-230.

[15] Huang $N$ E, Shen Z, Long $S R$, et al. The empirical mode decomposition and the Hilbert spectrum for nonlinear and non-stationary time series analysis [J].Proceedings of the Royal Society of London A: Mathematical, Physical and Engineering Sciences. The Royal Society, 1998, 454(1971): 903-995.

[16] Frei M G, Osorio I. Intrinsic time-scale decomposition: time-frequency-energy analysis and real-time filtering of non-stationary signals [J].Proceedings of the Royal Society of London A: Mathematical, Physical and Engineering Sciences. The Royal Society, 2007, 463(2078): 321-342. 